\title{
Identifying Brain Activity Specifically Related to the Maintenance and Perceptual Consequence of Central Sensitization in Humans
}

\author{
Michael C. Lee, ${ }^{1,2}$ Laura Zambreanu, ${ }^{1}$ David K. Menon, ${ }^{2}$ and Irene Tracey ${ }^{1}$ \\ ${ }^{1}$ Centre for Functional Magnetic Resonance Imaging of the Brain (FMRIB), Department of Clinical Neurology and Nuffield Department of Anaesthetics, \\ University of Oxford, Oxford OX3 9DU, United Kingdom, ${ }^{2}$ Department of Medicine, Division of Anaesthesia, University of Cambridge, Cambridge CB2 2QQ, \\ United Kingdom
}

\begin{abstract}
Central sensitization (CS) refers to an increase in the excitability of spinal dorsal horn neurons that results from, and far outlasts the initiating nociceptive input. Here, functional magnetic resonance imaging was used to examine whether supraspinal activity might contribute to the maintenance of CS in humans. A crossover parametric design was used to distinguish and control for brain activity that is related to the consequence of increased pain experienced during CS. When the intensity of pain during CS and normal states were matched, only activity within the brainstem, including the mesencephalic pontine reticular formation, and the anterior thalami remained increased during CS. Further analyses revealed that activity in the isolated brainstem area correlated positively with the force of noxious stimulation only during CS, whereas activity in the isolated thalamic area was not modulated parametrically in either CS or normal states. Additionally, the mean activity in the isolated brainstem area was increased only during CS, whereas the mean activity in the isolated thalamic area was increased in both states, albeit less so in the normal state. Together, these findings suggest a specific role of the brainstem for the maintenance of CS in humans. Regarding brain areas related to the consequence of increased pain perception during CS, we found that only cortical activity, mainly in the primary somatosensory area, was significantly correlated with intensity of pain that was attributable to both the force of noxious stimulation used and state in which noxious stimulation was applied.
\end{abstract}

Key words: central sensitization; pain; hyperalgesia; capsaicin; fMRI; brainstem

\section{Introduction}

Central sensitization (CS) refers to an increase in the excitability of neurons in the spinal dorsal horn that results from and far outlasts the initiating nociceptive input because of injury (Ji et al., 2003). Data from animal studies reveal that the maintenance of spinal excitability relies on descending facilitation from brainstem reticular nuclei (Suzuki et al., 2004); however, evidence in humans is lacking. The cardinal symptom of CS is secondary mechanical punctate hyperalgesia (SMPH). SMPH is increased pain behavior in response to noxious punctate stimulation of normal skin adjacent to the site of injury (IASP, 1994).

SMPH is commonly found in patients with chronic pain (Baron, 2006) but may also be experimentally induced in healthy volunteers by treating the skin with capsaicin (LaMotte et al., 1991). Capsaicin, a vallinoid compound, activates TRPV-1 (transient receptor potential vanilloid-1) receptors that are richly localized to nociceptors (Caterina et al., 1997). Several psychophysical studies suggest that CS is responsible for SMPH induced by

Received June 9, 2008; revised Sept. 9, 2008; accepted Sept. 23, 2008.

This work was supported by the UK Medical Research Council (M.C.L., D.K.M.) and by a Beverley and Raymond Sackler studentship (M.C.L.). We thank Drs. Vishvarani Wanigasekera, Giandomenico lannetti, Markus Ploner, and Prof. Rolfe-Detlef Treede for helpful advice and technical assistance.

Correspondence should be addressed to Dr. Michael C. Lee, Centre for Functional Magnetic Resonance Imaging of the Brain, John Radcliffe Hospital, Headington, 0xford, 0X3 9DU, UK. E-mail: mlee@fmrib.ox.ac.uk.

D01:10.1523/JNEUROSCI.2638-08.2008

Copyright $\odot 2008$ Society for Neuroscience $\quad$ 0270-6474/08/2811642-08\$15.00/0 capsaicin in humans. Intraneural microstimulation that normally elicits nonpainful referred sensation, caused pain after the skin adjacent to the area of referred sensation was treated with capsaicin (Torebjork et al., 1992). Furthermore, local anesthesia before capsaicin treatment limits the flare but not the development of SMPH (Klede et al., 2003). It is also established that SMPH develops and far outlasts the immediate pain caused by capsaicin (LaMotte et al., 1991). Together, these human studies indicate that capsaicin induced CS is initiated, but not exclusively maintained by peripheral nociceptive input (Ziegler et al., 1999; Ji et al., 2003).

Nearly a decade of neuroimaging research has revealed that supraspinal activity is increased during mechanical hyperalgesia that is experimentally induced by capsaicin in healthy volunteers (Iadarola et al., 1998; Baron et al., 1999; Zambreanu et al., 2005; Mainero et al., 2007). Increased activity is found in the brainstem, the thalami, cerebellum, primary and secondary somatosensory cortices, insula and cingulate cortex. However, hyperalgesia implies that increased pain occurs with identical stimulation of hyperalgesic skin compared with normal skin. As such, the increased activity observed in many brain regions likely reflects the experience of increased pain that is the consequence of CS. Indeed, activity in the insula, anterior cingulate cortex and somatosensory cortices has been shown to increase with increasing intensity of pain perceived under normal (nonhyperalgesic) conditions. (Coghill et al., 1999; Bornhövd et al., 2002).

In this present study, functional magnetic resonance imaging 
(fMRI) was used to isolate supraspinal activity that is related specifically to the maintenance of CS induced by capsaicin in humans. A crossover parametric design was used to distinguish and control for brain activity that is due to the consequence of increased pain perception during CS.

\section{Materials and Methods}

\section{Subjects}

Fifteen healthy subjects (right-handed; 8 males; mean age, 28 years; age range, 24-37 years) volunteered and were recruited after their written informed consent and full approval of the study by the Oxfordshire Local Ethics Committee (CO2-286).

\section{Study design}

The study used a crossover design to investigate pain perception and brain responses related to noxious mechanical stimulation of the skin during capsaicin induced CS and the normal state.

\section{CS and control sessions}

All subjects underwent two randomly ordered experimental sessions separated by $5-7 \mathrm{~d}$. In one session, capsaicin $(50 \mu \mathrm{g}$ dissolved in $0.1 \mathrm{ml}$ of Tween 80 ) was injected intradermally, $\sim 15 \mathrm{~cm}$ above the right medial malleolus. The site of injection was chosen because it was readily accessible during scanning and larger areas of hyperalgesia develop after capsaicin application in the lower limb compared with upper limb (Liu et al., 1998). Immediately after capsaicin injection, subjects rated the intensity of burning pain after injection over a five-min period using a numerical rating scale ( 0 , no pain; 100 , intolerable pain). Twenty minutes later, the presence of SMPH was tested within a $2 \mathrm{~cm}^{2}$ area drawn $5 \mathrm{~cm}$ distal to the site of injection. All subjects were also asked whether punctate stimulation in that area resulted in a greater intensity of sharp or prickling sensation when compared with similar stimulation of the homologous area in the left leg. The drawn area was designated for testing during the fMRI scan. All subjects were scanned $\sim 30$ min after capsaicin injection, which is when the area of SMPH is fully developed (LaMotte et al., 1991).

In the control session, the skin was left untreated. An area identical to where SMPH was tested in the capsaicin-treated session was drawn and designated for testing during the fMRI scan. Sham injection was not performed in the control session, because the aim of the study was not to ascertain whether capsaicin causes hyperalgesia (because this is already well accepted) but rather to examine the phenomenon of hyperalgesia that is established by capsaicin.

\section{Experimental paradigm}

During fMRI scanning, the skin designated for testing was stimulated with four punctate probes. Each probe delivered a different force (64, 128,256 , and $512 \mathrm{mN}$ ). The probes were cylindrical stainless-steel wires (tip diameter, $200 \mu \mathrm{m}$ ) mounted on rods of different brass weights. The rods moved ad libitum within a hand-held aluminum tube. Each probe was applied 12 times as earlier published psychophysical studies were able to demonstrate the left-ward shift in stimulus response function related to capsaicin induced hyperalgesia, using between five to 10 trials per category of force (Ziegler et al., 1999). Additionally, pilot and published studies from our laboratory indicate that 12 repeats provides adequate signal-to-noise ratio for measuring brain activity using fMRI (Zambreanu et al., 2005).

The probes were applied for one second each in pseudorandom sequence by the same researcher. After every stimulus, the subject used a computerized visual analog scale (VAS) to indicate the intensity of sharp sensation experienced (left anchor, not at all; right anchor, intolerable). The interstimulus interval was randomly jittered (between 28 and $32 \mathrm{~s}$ ) to allow higher effective sampling of the blood oxygenation level dependent (BOLD) signal after stimulation. The VAS appeared $12 \mathrm{~s}$ after each stimulus for a period of $6 \mathrm{~s}$.

\section{fMRI data acquisition}

fMRI data were acquired using a 3T Varian-Siemens whole-body magnetic resonance scanner. A head-only gradient coil was used with a birdcage radiofrequency coil for pulse transmission and signal reception. A whole-brain (including the midbrain, pons, and cerebellum) gradient- echo, echo-planar-imaging sequence was used for functional scans (echo time, $30 \mathrm{~ms}$; 43 contiguous 3 -mm-thick slices; field of view, $192 \times 192$ $\mathrm{mm}$; matrix $64 \times 64)$ with a repetition time of $3 \mathrm{~s}>495$ volumes. The first four volumes were discarded to permit equilibration of the BOLD signal. A T1-weighted $1 \mathrm{~mm}^{3}$ structural image was acquired for the registration of statistical activation maps.

\section{Data analysis}

Psychophysics. Subjects were considered to have developed SMPH if punctate stimulation of the capsaicin-treated area resulted in a subjective report of greater intensity of sharp or prickling sensation when compared with similar stimulation of the contra-lateral homologous area, and a statistically significant increase in VAS scores attributable to capsaicin treatment was demonstrated. The main effects of capsaicin treatment and force of punctate stimulation were tested in each individual using repeated measure ANOVA.

$f M R I$. All analyses were performed using FEAT (FMRIB Expert Analysis Tool) Version 5.67, part of FSL (FMRIB's Software Library; www. fmrib.ox.ac.uk/fsl). The following prestatistics were applied: motion correction, nonbrain removal, spatial smoothing using a Gaussian kernel of full-width, half-maximum, $5 \mathrm{~mm}$; grand-mean intensity normalization of the entire four-dimensional dataset by a single multiplicative factor; high-pass temporal filtering (Gaussian-weighted least-squares straight line fitting, with $\sigma=50 \mathrm{~s}$ ). Time-series statistical analysis was performed with local autocorrelation correction.

The input stimulus functions related to punctate stimulation with each force [(in $\mathrm{mN}) 64,128,256,512]$ and the task of rating were convolved with the gamma hemodynamic function (mean lag, $6 \mathrm{~s}$; full-width at half-height, $6 \mathrm{~s}$ ) to yield regressors in a general linear model that described the BOLD activity in the fMRI time-series. Parameter estimates (PE) were computed for the regressors that described BOLD activity associated with each force of punctate stimulation. These parameter estimates were also averaged to obtain a PE for the overall effect of punctate stimulation on BOLD activity.

All group analyses were performed using FLAME I (FMRIB Local Analysis of Mixed Effects) (Woolrich et al., 2004). For the purpose of group analyses, registration of low-resolution functional images to the corresponding high-resolution structural images was performed, followed by registration to a standard brain [Montreal Neurological Institute (MNI)-152 template] (Jenkinson et al., 2002). $Z$ score $>2.3$ was chosen as the significant threshold for the $Z$-statistic images from the group analysis. A cluster based method $(p<0.01)$ was used to correct for multiple comparisons (Friston et al., 1994).

The following hypothesis-driven analyses were performed.

The group-averaged PE that represented the increase in BOLD activity associated with punctate stimulation for each force [(in $\mathrm{mN}) 64,128$, $256,512]$ during both hyperalgesic and normal states were determined.

As with previous neuroimaging studies, the PE representing the overall effect of punctate stimulation on BOLD activity during the hyperalgesic state compared with the normal state was determined by a paired test.

The PEs for the punctate force in the normal and hyperalgesic state that produced a similar intensity of pain (the least difference in VAS ratings) was selected for each individual subject (see Fig. 1) and compared using a paired test. This analysis controlled for the increased pain intensity perceived during hyperalgesia and allowed the isolation of the supraspinal area where BOLD activity was specifically related to the process of CS.

Finally, a correlation analysis was performed to determine where BOLD activity correlated with the perception of pain intensity induced by either the state change or increasing force within states. The VAS scores, representing the perception of pain intensity associated with each punctate force that was applied in the normal and hyperalgesic states, were averaged across all subjects. These scores were demeaned and used as the covariate of interest in the analysis. After accounting for the mean effect of punctate stimulation across normal and hyperalgesic states, the BOLD activity that correlated with VAS scores, either positively or negatively, was identified. 

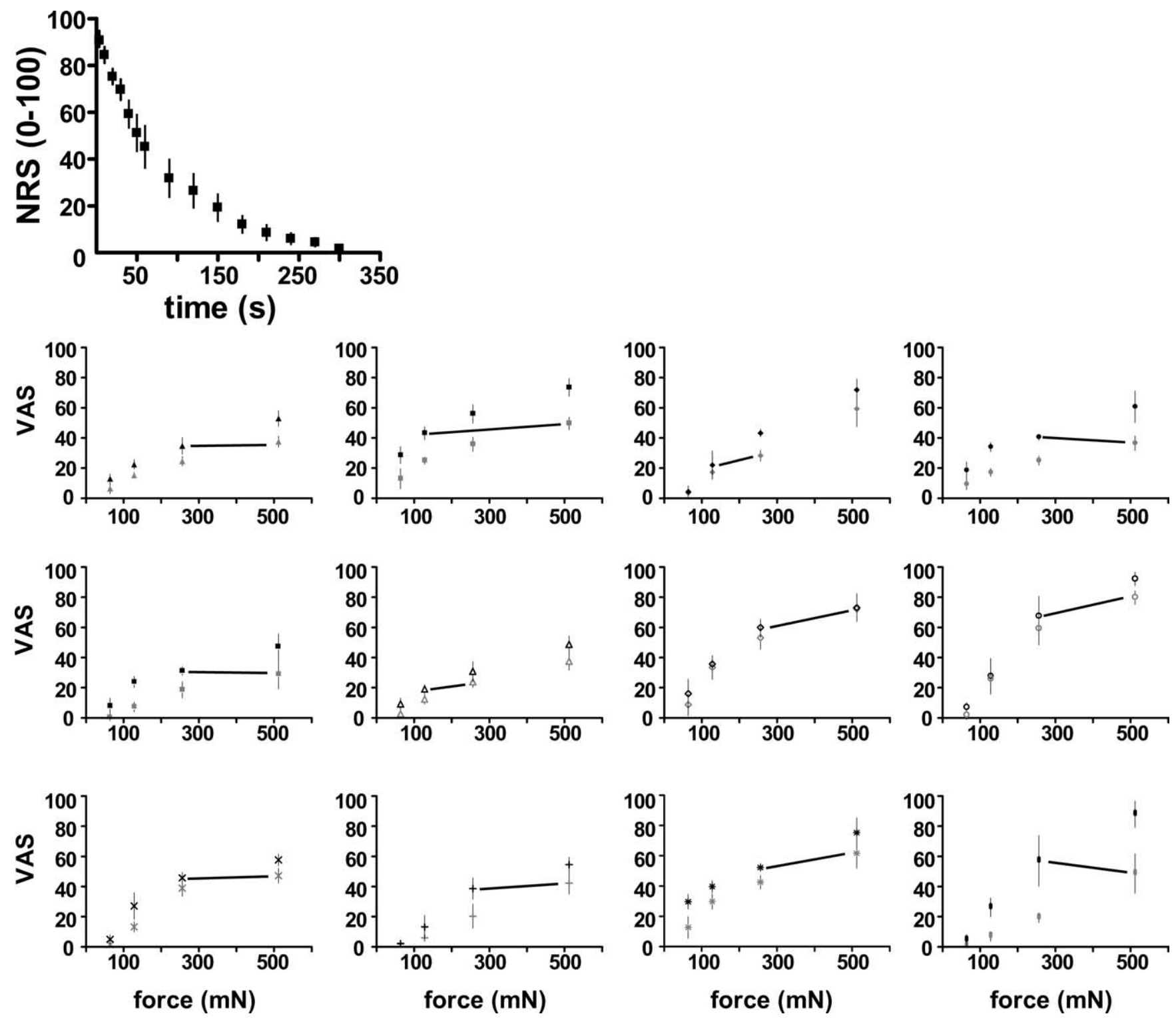

Figure 1. Top, Mean (SD) numeral rating scale (NRS) pain intensity scores over time after intradermal capsaicin. Bottom, Psychophysical stimulus response functions for punctate stimulation of the control (gray) and capsaicin-injected (black) sites for 12 subjects. Each subject is represented by a different symbol. The mean (SD) VAS scores of pain intensity that was associated with each force are plotted. There was a significant effect of force and capsaicin in all subjects $(p<0.01)$. The pair of forces adjoined by the black line had means that were least different.

\section{Results}

Psychophysical

All subjects experienced intense burning pain immediately after intradermal injection of capsaicin. The burning pain subsided within 5 to $10 \mathrm{~min}$ (Fig. 1, top graph). Twelve (right-handed, 6 males) developed SMPH using our criterion, as follows: an increased intensity of sensation related to the punctate stimulation of the area surrounding the injection site, compared with the homologous contra-lateral area, and a significant main effect of capsaicin on VAS-scores in each subject $(p<0.05)$ (Fig. 1, bottom graphs). The mean VAS-scores associated with punctate stimulation of $64,128,256$, and $512 \mathrm{mN}$ force from each subject were used in a group analysis (repeated measure ANOVA). As expected, there were significant main effects of capsaicin $(p<$ $\left.0.0001, F_{(1,50)}=63.16\right)$ and force $\left(p<0.0001, F_{(3,50)}=32.67\right)$ (Fig. 2, bottom graph). The posttests comparing VAS-scores related to stimulation with equal forces during capsaicin and control sessions, adjusted for multiple comparisons (Bonferroni's correction), were as follows: $64 \mathrm{mN}, p>0.05 ; 128 \mathrm{mN}, p<0.05$; $256 \mathrm{mN}, p<0.001 ; 512 \mathrm{mN}, p<0.001$.

\section{Imaging}

The aim of imaging in the present study was to investigate the neural correlates of CS. Therefore, only imaging data from the 12 subjects in whom CS was evidenced by SMPH were included in the analyses.

In the normal state, punctate stimulation increased BOLD activity in the bilateral anterior cingulate, anterior and posterior insular cortices, secondary somatosensory and supplemental motor areas and the contra-lateral primary somatosensory areas. A similar pattern of increase in BOLD activity was observed during hyperalgesia. However, there were additional increases of BOLD activity found in subcortical structures; namely the brainstem, the thalami, and cerebellum (Figs. 2, top; 3, top row).

The difference in the overall BOLD activity because of punctate stimulation between the hyperalgesic and normal states (not 


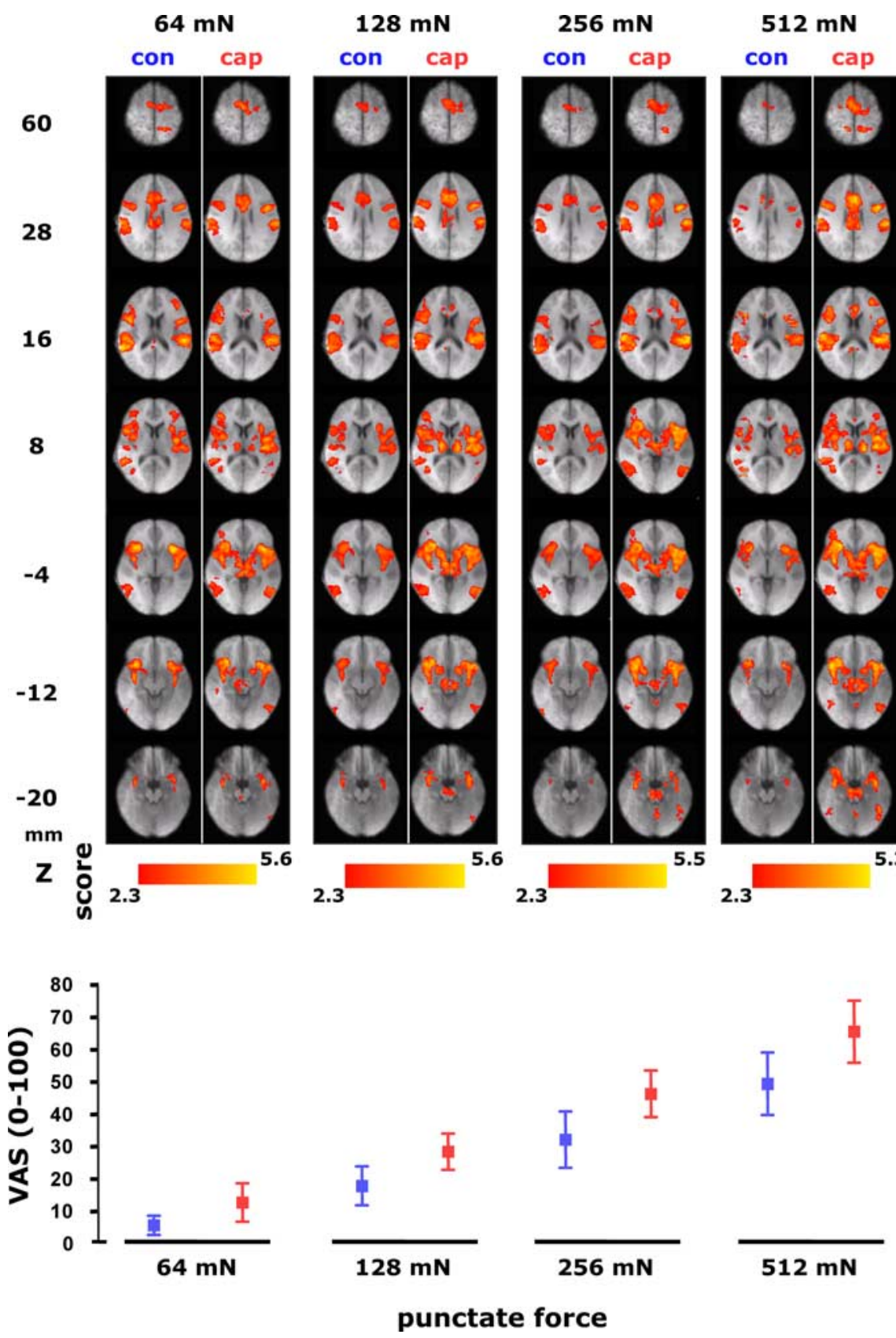

Figure 2. Top, Group-averaged activation maps for punctate forces [(in mN) 64, 128, 256, 512] for control (con) and hyperalgesic state (cap). Maps are displayed as axial slices (MNI Z-coordinates) along a vertical strip. Activity in brainstem and thalamus were significant only in the hyperalgesic state. Bottom, Group mean (SD) VAS scores of pain intensity associated with punctate forces [(in mN) 64, 128, 256, 512] in the control (blue) and capsaicin-treated (red) areas. There was a significant effect of force and capsaicin on the group mean VAS scores of pain intensity (repeated-measures ANOVA; main effects $p<0.01$; no interaction).

controlling for perception) was significant in several brain regions (Fig. 3, top row) (supplemental Table 1, available at www. jneurosci.org as supplemental material), including the brainstem thalami, left basal ganglia (caudate and putamen), amygdala, posterior insula, secondary somatosensory area, supplemental motor and primary somatosensory areas. Prefrontal areas that include the right frontal-polar and orbital-frontal cortices also showed increased activity.

Identification of subcortical activity related to the maintenance of $\mathrm{CS}$

When punctate stimulation was matched for the perception of pain in the hyperalgesic and normal states, the brainstem and thalami were the only areas where activity remained significantly increased in the hyperalgesic state compared with the normal state (Fig. 3, bottom row). Closer inspection of these areas revealed two noncontiguous clusters of activity.

The first cluster mainly includes the anterior thalami. The precise thalamic nuclei involved cannot be ascertained given the spatial resolution of the present wholebrain fMRI study. In fact, boundaries between thalamic nuclei that are visualized histologically are not easily seen in magnetic resonance images, even using contrast-optimized protocols (Magnotta et al., 2000). Nonetheless, the likely cortical connections associated with a given thalamic coordinate in MNI-space may be inferred through the use of MR atlases that provide the probabilities of cortical connections based on diffusion tractographic studies in humans (Behrens et al., 2003; Johansen-Berg et al., 2005). Using the Thalamic Connectivity Atlas implemented in FSLView 4.0 (www.fmrib.ox.ac.uk/fsl), voxels in anterior thalamic cluster were assigned to either the left or right thalamus, and the dominant cortical connection of each voxel was determined. We found that the dominant cortical connection was to the prefrontal cortex (PFC) for the majority of voxels, 73 and $56 \%$ for the right and left thalamus, respectively (supplemental Fig. 1, available at www.jneurosci.org as supplemental material).

The second cluster of activity was found within the brainstem. Attribution of fMRI activity to specific brainstem nuclei is possible but ideally requires acquisition protocols that are optimized for, and therefore limited to the brainstem (Dunckley et al., 2005). However, as the primary of aim of our study was to characterize activity in all supraspinal areas, rather than just within the brainstem, a whole-brain fMRI acquisition protocol was chosen that gives lower spatially resolved activity within the brainstem. In our study, the delineation of brainstem activity therefore relied on visual comparison with schematic drawings of gross anatomy obtained from a detailed atlas of the human brainstem (Duvernoy, 1995), because a template, stereotaxic brain, such as the atlas by Talairach and Tournoux (Talairach and Tournoux, 1988), does not exist for the brainstem. Visual inspection revealed that the brainstem activity occurred in several areas that included the mesencephalic pontine reticular formation (MPRF), along with the ventral tegmental area, substantia nigra, and red nucleus (Fig. 4). Notably, the increased activity in the MPRF appeared contralateral to the site of punctate stimulation, and unlike results of the earlier analysis that does not control for pain perception (Fig. 3, top row) (supplemental Table 1, available at www.jneurosci.org as supplemen- 

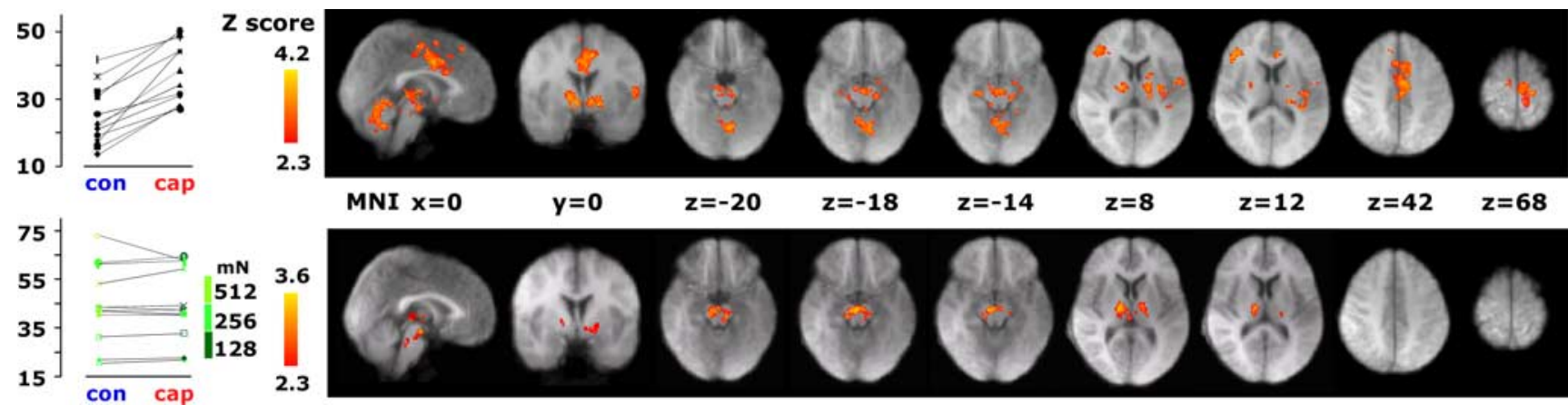

Figure 3. Top row, left, VAS scores of pain intensity (averaged across all forces) for each subject (represented by different symbols) during punctate stimulation in control (con) and capsaicin-treated areas (cap). Right, Paired comparison showing increased activity in the hyperalgesic state compared with the normal state for the overall effect of force. Bottom row, left, Punctate forces (represented by color) with the least different VAS scores during control and capsaicin sessions for each subject. Each subject is represented by the symbol previously used in Figure 1. Right, Paired comparison of the corresponding activation maps showing increased activity in the hyperalgesic state compared with the normal state. The MNI coordinates for each brain slice are indicated between the top and bottom rows.

tal material), activity in the periaqueductal gray (PAG) activity was not increased.

\section{Functional characteristics of subcortical activity related to the} maintenance of CS

To investigate whether thalamic and brainstem clusters were functionally similar, we examined the correlations between the percentage changes in the BOLD signal of both clusters during punctate stimulation and the force of punctate stimulation. This was performed for data obtained during CS and normal states. The percentage signal change was calculated as the mean of nonzero voxels in the binarized mask of the relevant cluster after affine transformation to functional space (Jenkinson et al., 2002).

We found that activity in the brainstem cluster increased with increasing force only during CS (linear regression; $1 /$ slope $=3199 ; r^{2}$ $=0.09, p=0.03)$ (Fig. 5 , top). A similar correlation of activity in the brainstem cluster was not observed in the normal state.

In contrast, activity in the thalamic cluster was increased in both hyperalgesic and normal states, albeit less so in the latter state. (Fig. 5, bottom, right). Also, unlike activity in the brainstem cluster, activity in thalamic cluster was uncorrelated with force in either hyperalgesic or normal state.

\section{Identification and characterization of cortical activity related to the perpetual consequence of CS}

We found that only cortical activity correlated positively with the intensity of pain induced by either the change in state or increased force used within states. Whereas activity in the primary motor area and the anterior cingulate cortex (ACC) correlated positively with perception of pain, the peak correlation $(Z$ score $=4.1)$ was found for activity in the contralateral primary somatosensory cortex (SI) (MNI coordinates, $-12,-40,72$ ) (Fig. 6).

To further delineate the cortical areas identified above in terms of their role in the perception of pain intensity, we examined whether activity in these areas also correlated with the intensity of pain perceived on a trial-by-trial basis. The correlations were examined for data obtained in the normal state as well as during CS. The correlations for each subject were computed using regressors that modeled brain activity based on the sequence of VAS ratings associated with each and every punctate stimulus. These regressors reflect the perception of pain intensity on a trialby-trial basis and are unique to each subject and the state in which pain was perceived by that subject (Davis et al., 2002). Brain activity associated with punctate stimulation and the task of pain rating were modeled as confound regressors. The group mean correlations were then generated for the normal state and as well as during CS. All analyses used statistical methods that had been described earlier. An uncorrected voxel-based threshold, Z score $>2.3$ was used to identify correlations within the cortical area of interest. For both the CS and normal states, we found significant correlations only for activity within the right lower limb representation of SI (Fig. 6).

\section{Discussion}

Our results suggest that brainstem activity contributes to the maintenance of the CS state in humans whereas activity in the somatosensory cortex reflects the perceptual consequence of CS that is an increase in the intensity of pain experienced.

\section{A specific role for the brainstem in the maintenance of central sensitization}

Several imaging laboratories, including ours, have mapped widespread increases in brain activity to an identical force of punctate stimulation in the hyperalgesic state compared with the normal state (Baron et al., 1999; Zambreanu et al., 2005). However, amplification of input from peripheral mechanosensitive afferents occurs at the level of the spinal dorsal horn (Sun et al., 2004) before further transmission to supraspinal areas. The increased activity in supraspinal areas observed in earlier neuroimaging studies during hyperalgesia might therefore be related to the consequence of spinal amplification of nociceptive input (i.e., increased pain perception). This interpretation is consistent with our finding that the increases in brain activity were widespread when identical punctate stimulation during the hyperalgesic and normal states were compared. (Figs. 2; 3, top row). Yet evidence from these studies, particularly our own (Iannetti et al., 2005; Zambreanu et al., 2005), do suggest that the increased brainstem activity during hyperalgesia reflects CS maintaining mechanisms, such as descending facilitation, as shown in animal studies. However, because of confounds related to perception the data to date is equivocal.

To control for the epiphenomenon of pain perception and thereby determine supraspinal areas specific to the maintenance of CS, we compared BOLD activity related to punctate stimulation that was associated with similar pain ratings in the normal and hyperalgesic states. We found that brainstem activity in the hyperalgesic state remained increased compared with the normal state even after the perception of pain was matched in both states. Because the intensity of pain was similar in both states, and for the hyperalgesic state resulted from a less forceful punctate stim- 

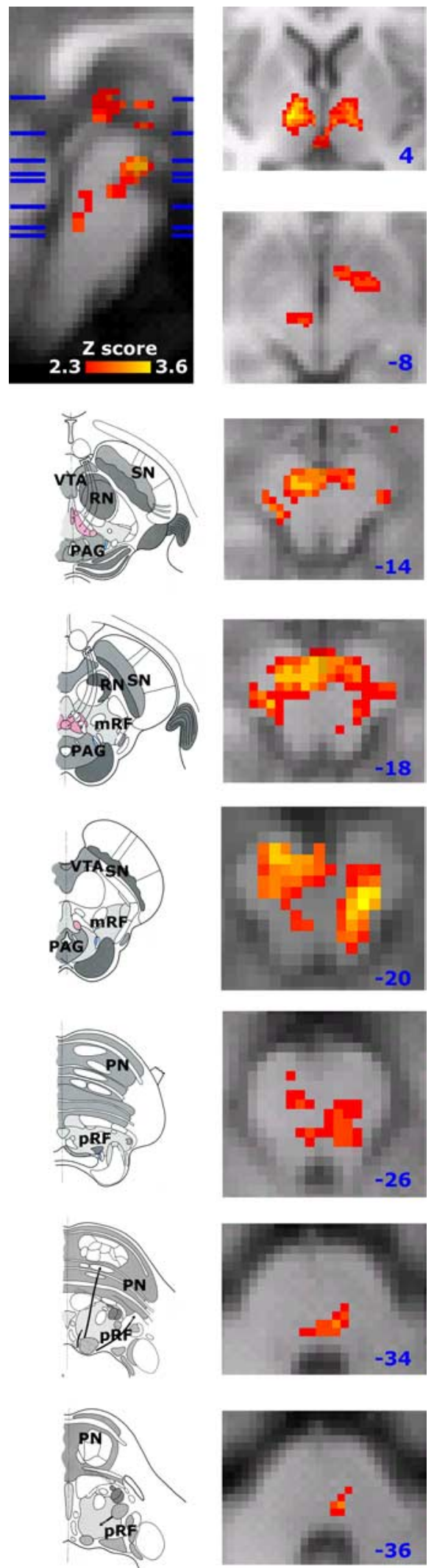

Figure 4. Localization of the clusters showing increased activity in the hyperalgesic state compared with the normal state. The right, top-most image shows where activity is increased in the midsaggital section of the brainstem and thalamic areas. Blue lines indicate where the axial slices are positioned. The MNIZ-coordinate for each axial slice is indicated in blue. Annotated diagrams (Duvernoy, 1995) that correspond to each axial slice are included to guide anatomical localization in the brainstem. VTA, Ventral tegmentum area; SN, substantia nigra; RN, red nucleus; mRF, mesencephalic reticular formation; pRF, pontine reticular formation; $\mathrm{PN}$, pontine nuclei.
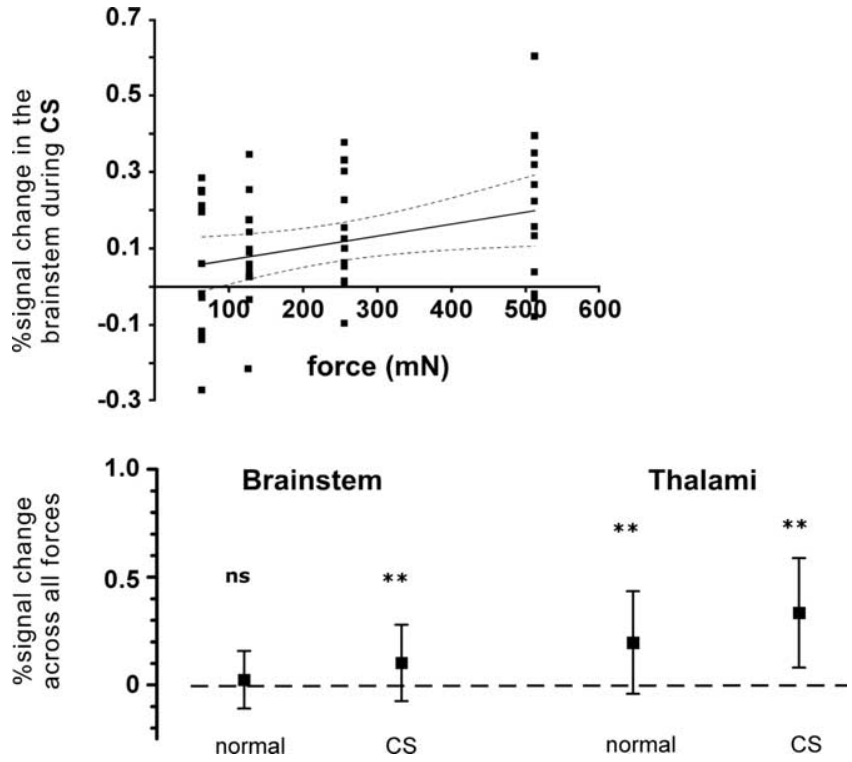

Figure 5. Top, The percentage of BOLD signal change in the brainstem increased significantly with increasing force in the hyperalgesic state only ( $p=0.03 ; 95 \%$ confidence intervals shown). Bottom, The mean percentage of BOLD signal change in the brainstem during punctate stimulation across all forces was not significantly different $(p=0.1)$ from zero in the normal state. Two-tailed, one sample $t$ tests were performed. Means and SDs are shown. ns, Nonsignificant. ${ }^{* *} p<0.0001$.

ulation, neither nociceptive input nor perception adequately accounts for the increased activity in the brainstem. We propose that this increase in activity represents the supraspinal modulation of spinal processing which leads to the eventual facilitation of nociceptive input and the maintenance of CS.

Our proposal is supported by the observation that the increased brainstem activity was localized to areas that included the MPRF (Fig. 3). The MPRF itself consists of the nucleus cuneiformis reticularis and pontine reticular nuclei. The role of these nuclei in the supraspinal facilitation of nociceptive processing is well recognized in animal studies (Zhuo and Gebhart, 1992, 2002). They receive a large afferent input from nociceptive lamina I dorsal horn neurons, project directly to the rostral ventral medial medulla (RVM) (Zemlan and Behbehani, 1988), and possess nocifensive reflex-related neurons with neurophysiological characteristics similar to those reported in the RVM (Carlson et al., 2005). The RVM is an integral part of the brainstem reticular formation that facilitates nociceptive processing at the level of the spinal dorsal horn during CS (Gebhart, 2004). After tissue injury, CS is initiated in the spinal dorsal horn. Input from the spinal dorsal horn ascends from the spinal dorsal horn to the MPRF (Mantyh et al., 1997). Output from nuclei in the MPRF activates descending pathways from the RVM that enhances nociceptive processing at the level of the spinal dorsal horn thereby completing a facilitatory spinal-bulbar circuit that maintains CS (Suzuki et al., 2002). Descending facilitatory pathways are not demonstrably involved during nociceptive processing in the normal state (Ossipov and Porreca, 2006). However, other brainstem nuclei, for example those involved in nociceptive transmission or autonomic response, may activate in response to ascending spinal nociceptive input (Petrovic et al., 2004; Dunckley et al., 2005). Hence, the absence of increased activity in the brainstem area identified as the MPRF during nociceptive processing in the normal state (Fig. 4, bottom, left graph), further supports its specific role in CS. In contrast, the increase in thalamic activity in response to punctate stimulation across all forces was present (albeit reduced) in the normal state (Fig. 4, bottom, right graph). 


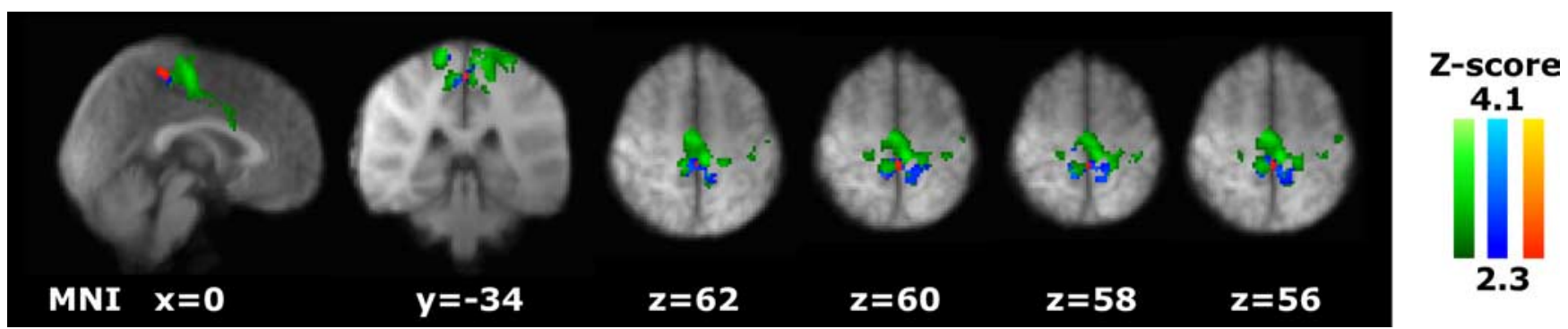

Figure 6. The brain areas in which activity correlated positively with the perception of pain intensity across the normal and CS states are shown in green. Within these areas, activity in the contralateral primary somatosensory correlated with perception of pain intensity associated with punctate stimulation during $C S$ (shown in red) and in the normal state (shown in blue).

Facilitatory brainstem activity during central sensitization The isolated brainstem activity might represent either a facilitatory influence or the release of tonic inhibition on nociceptive processing at the level of the spinal dorsal horn or both. This cannot be fully ascertained without selective pharmacological inactivation of the brainstem area where activity was found.

Nonetheless, we argue for a facilitatory role for the brainstem activity isolated in our study for the following reasons. The release of tonic inhibition by the brainstem would have been suggested had the BOLD responses been greater in the normal rather than the hyperalgesic state. Such activity would likely be localized to the PAG that plays an established role in the descending inhibition of spinal nociceptive processing. However, in our study, the identified brainstem activity was clearly greater during the hyperalgesic state and localized to the MPRF rather than to the PAG. Additionally, activity in the isolated brainstem area correlated positively with the force of noxious stimulation during CS. The correlation between nociceptive input and brainstem activity during CS resembles the electrophysiological response of "on-cells" that are nocimodulatory and a source of descending facilitation (Hernández and Vanegas, 2001). Although the force of stimulation only accounted in part for the variation in brainstem activity observed during CS, similar correlations were not observed in the brainstem during the normal state, or in the thalami for either state.

\section{A role for the anterior thalamus and PFC in the cognitive control of hyperalgesia}

Only the sensory-discriminatory aspect of pain was matched between normal and hyperalgesic states in this study. As such, the increased BOLD activity isolated after controlling for the sensory dimension of pain may reflect differences related to other aspects of a painful experience during CS that were not assessed in the study, for example its affective or motivational aspects (Price, 2000).

Stress attributable to a painful injection of capsaicin can influence how the nervous system processes subsequent nociceptive stimuli. However, in our study, pain attributable to the capsaicin injection was very short-lived (Fig. 1, top), and its effects are unlikely to carry over to the imaging period (Lewis et al., 1980). For example, in our pilot psychological study, scores from State Anxiety questionnaires (Spielberger and Gorsuch, 1983) that were collected after capsaicin injection did not significantly differ from those collected in sessions in which no injection was administrated (supplemental Fig. 2, available at www.jneurosci.org as supplemental material). It should also be noted that acute stress is usually associated with analgesia and not hyperalgesia (Willer et al., 1981). Therefore, the effects of stress cannot account for hyperalgesia and the increase of BOLD activity observed in our study during CS.

However, pain that is experienced in the context of previous injury (capsaicin injection) is more salient and may be more thoroughly appraised by the subject. However, the mechanisms underlying the cognitive appraisal of pain should also operate to some extent during pain experienced in the normal state. Because the increased brainstem activity isolated in our study was absent in the normal state, the brainstem activity is probably unrelated to the cognitive appraisal of pain. In fact, the cognitive appraisal of pain is thought to be substantiated by activity in the dorsolateral and ventral-lateral PFC (Wiech et al., 2008). Indeed, the increased PFC activity that is consistently observed during neuroimaging studies of hyperalgesia (including ours) has been attributed to such increased cognitive appraisal during hyperalgesia (Lorenz et al., 2002). PFC activity has also been shown to exert an inhibitory influence on nociceptive processing through modulation of thalamicmidbrain connectivity (Lorenz et al., 2003). Given the likely cortical connections of the anterior thalami to the PFC, and that increased activity in both was observed during CS, we speculate that the anterior thalamic activity represented part of the cortical control of nociceptive processing driven by the increased PFC activity during CS.

\section{Increased pain intensity as the perceptual consequence of} central sensitization is reflected by increased cortical activity Neuroimaging and electrophysiological studies in humans have long demonstrated that activity in cortical areas such as SI and ACC encodes the perception of pain in the normal state (Rainville et al., 1997; Coghill et al., 1999; Timmermann et al., 2001; Bornhövd et al., 2002; Gross et al., 2007). In this present study, activity in SI and ACC was correlated positively with the intensity of pain that was attributable to both the force of noxious stimulation used and state in which noxious stimulation was applied. Thus, activity in SI and ACC are more related to the intensity of pain perception rather than to the force of noxious stimulation or the state in which noxious stimulation was delivered. Furthermore, SI activity was significantly correlated with the intensity of pain that was experienced on a trial-by-trial basis in the normal state as well as during CS. These findings suggest that the increased cortical activity found in SI and ACC in previous neuroimaging studies of secondary mechanical hyperalgesia (Iadarola et al., 1998; Baron et al., 1999; Zambreanu et al., 2005; Mainero et al., 2007) mainly reflects the increased intensity of pain experienced during CS.

\section{Conclusion}

We used a novel experimental approach to functionally dissect the increased brain activity found during CS. Our results suggest that brainstem activity maintains CS in humans whereas cortical activity, particularly in the SI, reflects the increased intensity of pain experienced during CS. Although further work that provides unequivocal localization of brainstem activity involved in CS is required, these results translate research findings from preclinical models of CS to humans and may inform the development of a noninvasive biomar- 
ker of CS that can be used to identify potential anti-hyperalgesics in humans (Iannetti et al., 2005; Tracey and Mantyh, 2007).

\section{References}

Baron R (2006) Mechanisms of disease: neuropathic pain-a clinical perspective. Nat Clin Pract Neurol 2:95-106.

Baron R, Baron Y, Disbrow E, Roberts TP (1999) Brain processing of capsaicin-induced secondary hyperalgesia: a functional MRI study. Neurology 53:548-557.

Behrens TE, Johansen-Berg H, Woolrich MW, Smith SM, Wheeler-Kingshott CA, Boulby PA, Barker GJ, Sillery EL, Sheehan K, Ciccarelli O, Thompson AJ, Brady JM, Matthews PM (2003) Non-invasive mapping of connections between human thalamus and cortex using diffusion imaging. Nat Neurosci 6:750-757.

Bornhövd K, Quante M, Glauche V, Bromm B, Weiller C, Büchel C (2002) Painful stimuli evoke different stimulus-response functions in the amygdala, prefrontal, insula and somatosensory cortex: a single-trial fMRI study. Brain 125:1326-1336.

Carlson JD, Selden NR, Heinricher MM (2005) Nocifensive reflex-related on- and off-cells in the pedunculopontine tegmental nucleus, cuneiform nucleus, and lateral dorsal tegmental nucleus. Brain Res 1063:187-194.

Caterina MJ, Schumacher MA, Tominaga M, Rosen TA, Levine JD, Julius D (1997) The capsaicin receptor: a heat-activated ion channel in the pain pathway. Nature 389:816-824.

Coghill RC, Sang CN, Maisog JM, Iadarola MJ (1999) Pain intensity processing within the human brain: a bilateral, distributed mechanism. J Neurophysiol 82:1934-1943.

Davis KD, Pope GE, Crawley AP, Mikulis DJ (2002) Neural correlates of prickle sensation: a percept-related fMRI study. Nat Neurosci 5:1121-1122.

Dunckley P, Wise RG, Fairhurst M, Hobden P, Aziz Q, Chang L, Tracey I (2005) A comparison of visceral and somatic pain processing in the human brainstem using functional magnetic resonance imaging. J Neurosci 25:7333-7341.

Duvernoy H (1995) The human brain stem and cerebellum. Surface, structure, vascularization and three-dimensional anatomy with MRI. Wien: Springer.

Friston KJ, Worsley KJ, Frackowiak RSJ, Mazziotta JC, Evans AC (1994) Assessing the significance of focal activations using their spatial extent. Hum Brain Mapp 1:214-220.

Gebhart GF (2004) Descending modulation of pain. Neurosci Biobehav Rev 27:729-737.

Gross J, Schnitzler A, Timmermann L, Ploner M (2007) Gamma oscillations in human primary somatosensory cortex reflect pain perception. PLoS Biol 5:e133.

Hernández N, Vanegas H (2001) Encoding of noxious stimulus intensity by putative pain modulating neurons in the rostral ventromedial medulla and by simultaneously recorded nociceptive neurons in the spinal dorsal horn of rats. Pain 91:307-315.

Iadarola MJ, Berman KF, Zeffiro TA, Byas-Smith MG, Gracely RH, Max MB, Bennett GJ (1998) Neural activation during acute capsaicin-evoked pain and allodynia assessed with PET. Brain 121:931-947.

Iannetti GD, Zambreanu L, Wise RG, Buchanan TJ, Huggins JP, Smart TS, Vennart W, Tracey I (2005) Pharmacological modulation of painrelated brain activity during normal and central sensitization states in humans. Proc Natl Acad Sci U S A 102:18195-18200.

IASP (1994) Part III: Pain terms, a current list with definitions and notes on usage. In: Classification of chronic pain. Descriptions of chronic pain syndromes and definitions of pain terms, Ed 2. (Merskey M, Bogduk N, eds), pp 209-214. Seattle: IASP.

Jenkinson M, Bannister P, Brady M, Smith S (2002) Improved optimization for the robust and accurate linear registration and motion correction of brain images. Neuroimage 17:825-841.

Ji RR, Kohno T, Moore KA, Woolf CJ (2003) Central sensitization and LTP: do pain and memory share similar mechanisms? Trends Neurosci 26:696-705.

Johansen-Berg H, Behrens TE, Sillery E, Ciccarelli O, Thompson AJ, Smith SM, Matthews PM (2005) Functional-anatomical validation and individual variation of diffusion tractography-based segmentation of the human thalamus. Cereb Cortex 15:31-39.

Klede M, Handwerker HO, Schmelz M (2003) Central origin of secondary mechanical hyperalgesia. J Neurophysiol 90:353-359.

LaMotte RH, Shain CN, Simone DA, Tsai EF (1991) Neurogenic hyperalgesia: psychophysical studies of underlying mechanisms. J Neurophysiol 66:190-211.

Lewis JW, Cannon JT, Liebeskind JC (1980) Opioid and nonopioid mechanisms of stress analgesia. Science 208:623-625.
Liu M, Max MB, Robinovitz E, Gracely RH, Bennett GJ (1998) The human capsaicin model of allodynia and hyperalgesia: sources of variability and methods for reduction. J Pain Symptom Manage 16:10-20.

Lorenz J, Cross D, Minoshima S, Morrow T, Paulson P, Casey K (2002) A unique representation of heat allodynia in the human brain. Neuron 35:383-393.

Lorenz J, Minoshima S, Casey KL (2003) Keeping pain out of mind: the role of the dorsolateral prefrontal cortex in pain modulation. Brain 126:1079-1091.

Magnotta VA, Gold S, Andreasen NC, Ehrhardt JC, Yuh WT (2000) Visualization of subthalamic nuclei with cortex attenuated inversion recovery MR imaging. Neuroimage 11:341-346.

Mainero C, Zhang WT, Kumar A, Rosen BR, Sorensen AG (2007) Mapping the spinal and supraspinal pathways of dynamic mechanical allodynia in the human trigeminal system using cardiac-gated fMRI. Neuroimage 35:1201-1210.

Mantyh PW, Rogers SD, Honore P, Allen BJ, Ghilardi JR, Li J, Daughters RS, Lappi DA, Wiley RG, Simone DA (1997) Inhibition of hyperalgesia by ablation of lamina I spinal neurons expressing the substance P receptor. Science 278:275-279.

Ossipov MH, Porreca F (2006) Descending excitatory systems. In: Pain, Ed 3 (Cervero F, Jensen TS, eds), pp 193-218. Amsterdam: Elsevier.

Petrovic P, Petersson KM, Hansson P, Ingvar M (2004) Brainstem involvement in the initial response to pain. Neuroimage 22:995-1005.

Price DD (2000) Psychological and neural mechanisms of the affective dimension of pain. Science 288:1769-1772.

Rainville P, Duncan GH, Price DD, Carrier B, Bushnell MC (1997) Pain affect encoded in human anterior cingulate but not somatosensory cortex. Science 277:968-971.

Spielberger CD, Gorsuch RL (1983) Manual for the state-trait anxiety inventory (form Y) ("self-evaluation questionnaire"). Palo Alto, CA: Consulting Psychologists.

Sun RQ, Lawand NB, Lin Q, Willis WD (2004) Role of calcitonin gene-related peptide in the sensitization of dorsal horn neurons to mechanical stimulation after intradermal injection of capsaicin. J Neurophysiol 92:320-326.

Suzuki R, Morcuende S, Webber M, Hunt SP, Dickenson AH (2002) Superficial NK1-expressing neurons control spinal excitability through activation of descending pathways. Nat Neurosci 5:1319-1326.

Suzuki R, Rygh LJ, Dickenson AH (2004) Bad news from the brain: descending 5-HT pathways that control spinal pain processing. Trends Pharmacol Sci 25:613-617.

Talairach J, Tournoux P (1988) Co-planar stereotaxic atlas of the human brain. New York: Thieme Medical.

Timmermann L, Ploner M, Haucke K, Schmitz F, Baltissen R, Schnitzler A (2001) Differential coding of pain intensity in the human primary and secondary somatosensory cortex. J Neurophysiol 86:1499-1503.

Torebjork HE, Lundberg LE, LaMotte RH (1992) Central changes in processing of mechanoreceptive input in capsaicin-induced secondary hyperalgesia in humans. J Physiol 448:765-780.

Tracey I, Mantyh PW (2007) The cerebral signature for pain perception and its modulation. Neuron 55:377-391.

Wiech K, Ploner M, Tracey I (2008) Neurocognitive aspects of pain perception. Trends Cogn Sci 12:306-313.

Willer JC, Dehen H, Cambier J (1981) Stress-induced analgesia in humans: endogenous opioids and naloxone-reversible depression of pain reflexes. Science 212:689-691.

Woolrich MW, Behrens TE, Beckmann CF, Jenkinson M, Smith SM (2004) Multilevel linear modelling for FMRI group analysis using Bayesian inference. Neuroimage 21:1732-1747.

Zambreanu L, Wise RG, Brooks JC, Iannetti GD, Tracey I (2005) A role for the brainstem in central sensitisation in humans. Evidence from functional magnetic resonance imaging. Pain 114:397-407.

Zemlan FP, Behbehani MM (1988) Nucleus cuneiformis and pain modulation: anatomy and behavioral pharmacology. Brain Res 453:89-102.

Zhuo M, Gebhart GF (1992) Characterization of descending facilitation and inhibition of spinal nociceptive transmission from the nuclei reticularis gigantocellularis and gigantocellularis pars alpha in the rat. J Neurophysiol 67:1599-1614.

Zhuo M, Gebhart GF (2002) Facilitation and attenuation of a visceral nociceptive reflex from the rostroventral medulla in the rat. Gastroenterology 122:1007-1019.

Ziegler EA, Magerl W, Meyer RA, Treede RD (1999) Secondary hyperalgesia to punctate mechanical stimuli. Central sensitization to A-fibre nociceptor input. Brain 122:2245-2257. 